\title{
CAMPO ÉTNICO, POLOS DE CAPITAL Y ESTRATEGIAS. EL CASO DE LOS JÓVENES MARROQUÍES EN BIZKAIA
}

\author{
ETHNIC FIELD, CAPITAL POLES, AND STRATEGIES. THE CASE OF THE \\ MOROCCAN YOUNGSTER IN BIZKAIA
}

\author{
Mikel Barba del Horno \\ Universidad del País Vasco/ Euskal Herriko Unibertsitatea, Bizkaia / España \\ mikelbarba@gmail.com \\ http://orcid.org/0000-0002-4261-9843
}

Recibido/Received: 10/11/2019

Modificado/Modified: 16/04/2020

Aceptado/Accepted: 18/06/2020

\section{RESUMEN}

En el artículo se analizan las estrategias de incorporación de los jóvenes marroquíes en Bizkaia utilizando un marco teórico que se basa en la teoría de campos de Pierre Bourdieu. A través del análisis de una serie de entrevistas cualitativas se identifican los principales polos de capital cultural étnico que influyen en los jóvenes marroquíes y se analizan dos tipos ideales de estrategia de incorporación: el repliegue étnico y la asimilación. Los resultados muestran que la asimilación es una estrategia que proporciona un menor acceso a recursos materiales.

\section{PALABRAS CLAVE}

Capital cultural; capital social; incorporación de inmigrantes; menores extranjeros no acompañados.

\section{SUMARIO}

1. Introducción. 2. Teoría. 3. Metodología. 4. Resultados. 5. Conclusiones. Bibliografía.

\begin{abstract}
In this article we analyze the incorporation strategies of the Moroccan youngsters in the province of Bizkaia, using a theoretical approach based in Pierre Bourdieu's field theory. Through the analysis of an array of qualitative interviews we identify the ethnic cultural capital poles that influence in Moroccan youngsters and we define two ideal types of incorporation strategies: the ethnic retreat and the assimilation. The results show that the assimilation strategy offers less access to material resources.
\end{abstract}

\section{KEYWORDS}

Cultural capital; Social capital; Immigrant incorporation; Unaccompanied foreign minors.

\section{CONTENTS}

1. Introduction. 2. Theory. 3. Methodology. 4. Results. 5. Conclusions. References. 


\section{INTRODUCCIÓN}

El aparato teórico desarrollado por el sociólogo Pierre Bourdieu, proporciona una herramienta muy potente para estudiar conjuntamente diferencias culturales, desigualdad y conexiones sociales. Los conceptos de campo social, capital cultural, capital social o habitus fueron creados originalmente para explicar cómo las diferencias culturales entre diferentes clases sociales dan lugar a desigualdades. Aunque existen algunos ejemplos del empleo de la teoría de Bourdieu en el estudio de la etnicidad, las aplicaciones en este campo han sido mucho menores.

Con la intención de contribuir a cubrir una parte de este vacío, en este artículo se emplea un marco teórico elaborado a partir de los conceptos de Bourdieu parar analizar los procesos de incorporación de los jóvenes inmigrantes marroquíes en la provincia de Bizkaia (España). Hablamos de personas que han migrado como menores sin acompañamiento adulto y han sido tutelados por la administración. Encajarían, por lo tanto, en lo que se ha venido a denominar generación 1.5 aunque con una especificidad importante: sus procesos de incorporación están mediatizados por la actuación de las administraciones públicas que los acogen como menores.

La inmigración de Menores Extranjeros No Acompañados (MENAs) se inicia en Europa a mediados de la década de los noventa y se caracteriza por la llegada de inmigrantes menores de edad, fundamentalmente africanos, sin acompañamiento de adultos. Constituyen un problema específico para las administraciones porque se trata de un colectivo protegido por la legislación del menor y, por lo tanto, el Estado debe hacerse cargo de la tutela de estos menores.

En España y en la Comunidad Autónoma Vasca (CAV) las llegadas de los primeros MENAs se inician en los años 90 y alcanzan un pico en los últimos años de la primera década del 2000. Con la crisis económica que se inicia en 2008 se produce una reducción de las llegadas y, desde 2014 la recuperación económica ha dado lugar a un incremento espectacular de cerca del 3000\% en el período 2014-2018 (El diario vasco 9-9-2019).

La bibliografía existente en torno a los MENAs empezó abordando los temas de la descripción del colectivo (cuantificación, diferentes perfiles, etc.) y de la inserción en el sistema de acogida y los problemas y las vulneraciones de los derechos de los menores que ello generaba (Ararteko, 2005; Setién y Barcelo, 2007; Quiroga, Alonso y Soria, 2009; Gozalo, Jiménez y Vozmediano, 2010; Ararteko, 2011). Recientemente se han tratado temas con un mayor perfil sociológico como la construcción de la identidad de los menores en el contexto del sistema de acogida (Lajusticia, 2018), el aislamiento respecto a la población autóctona (Barba del Horno, 2019) o la percepción de los menores en los centros educativos (Belmonte, Meneses, Cano y Cabrera, 2019).

La acogida de MENAs en Bizkaia se ha realizado a través de una red de acogida diferenciada de la que acoge a los menores autóctonos. En esta red especializada en menores extranjeros la acogida se hace preferentemente a través de grandes centros, en ocasiones masificados, que se encuentran, por lo general, separados de los núcleos urbanos (Quiroga, Alonso y Soria, 2009). Esto dificulta que los menores puedan tener un proceso de socialización satisfactorio en la sociedad de acogida, por encontrarse aislados de los principales espacios de socialización normalizados (Barba del Horno, 2019). La inserción en el sistema educativo se produce también a través de un sistema no normalizado de centros orientados a la exclusión social o al fracaso escolar (Fernández y Barba del Horno, 2014).

Los problemas de convivencia que se han producido en diferentes centros de menores han dado el salto a la prensa contribuyendo a crear una imagen muy negativa del colectivo. 
Podríamos decir que los jóvenes marroquíes sufren uno de los estigmas más extremos dentro de la población inmigrante. El colectivo marroquí es el colectivo migrante que sufre una peor valoración por parte de la población autóctona que tiende a relacionarlo con la violencia, la delincuencia, las agresiones sexuales, etc. (Fernández, Valbuena y Caro, 2017; Ikuspegi, 2018). Este estigma da lugar a una variedad de prácticas discriminatorias que reducen la posibilidad de los jóvenes marroquíes de relacionarse con población autóctona: se les veta la entrada en bares y discotecas, se les identifica recurrentemente cuando pasean por la calle (Díaz y Fantova, 1998; Aranguren, 2011).

Por otro lado, cumplidos los dieciocho años y terminada la etapa de tutela, los jóvenes inmigrantes se exponen a una situación de gran vulnerabilidad (Palma-García, Ruiz Mosquera, y Vives González, 2019). En el caso de Bizkaia, algunos tienen la posibilidad de acceder a un programa de emancipación, Mundutik Mundura, que les garantiza una vivienda, unos ingresos mínimos y el acompañamiento de un educador. Los jóvenes que, en base a su proceso educativo, no consiguen acceder a este programa suelen terminar en una situación de desamparo que conduce, a menudo, a la indigencia.

\section{TEORÍA}

Partimos de un marco teórico basado en la teoría de campos de Pierre Bourdieu (Bourdieu, 2005), (Bourdieu, 1997), (Bourdieu, 1988), (Bourdieu, 1986) adaptada al estudio de las relaciones interétnicas. La noción de campo social de Bourdieu es útil para describir cómo los diferentes grupos sociales ponen en juego las diferencias culturales para generar desigualdad. En este sentido, las prácticas de los diferentes actores y grupos en el campo buscan la apropiación de un tipo determinado de capital, de poder, que les va a dar acceso a recursos. Estamos ante una visión materialista de las diferencias culturales.

En el campo étnico estaría en juego un capital cultural ligado la pertenencia étnica, a los repertorios culturales propios de cada grupo étnico: idioma, creencias, valores, rasgos de personalidad aceptados por el grupo, formas de expresión corporal, etc. A diferencia del concepto de campo de Bourdieu en el que existe un único capital cultural en juego en el campo social (el de la case dominante), proponemos un campo social en el que conviven diferentes capitales culturales vinculados a los diferentes grupos étnicos. Cada grupo étnico define un subcampo social en base a su identidad. De esta manera, cada capital cultural se generaría en torno a una identidad étnica específica. En las relaciones interétnicas asimétricas existiría un capital cultural dominante y una serie de capitales subculturales que corresponderían a las diferentes minorías étnicas. La distinción entre capitales culturales dominantes y no dominantes ha demostrado su utilidad en la investigación sociológica reciente (Carter, 2003).

Consideramos que los diferentes capitales culturales, las diferentes identidades, operan en un mismo campo porque las identidades étnicas se constituyen en sus relaciones con otras identidades (Barth, 1976). Es decir, cuando un grupo étnico define el contenido cultural significativo de su identidad, lo hace por comparación con los contenidos culturales de otros grupos étnicos, de manera que se suele otorgar una importancia mucho mayor a aquellos rasgos que son diferenciadores con otros grupos. Tomamos aquí el concepto de gramática propuesto por Bauman y Gingrich (2006), en base al cual la construcción de la identidad y la alteridad se producen de manera simultánea y se articulan en base a tres modelos/gramáticas; la orientalización que define al otro por oposición a uno mismo, la segmentación que define una identidad más amplia o más reducida según el contexto y el 
abarcamiento que incluye al otro como parte de un todo que es la sociedad, aunque en una posición subordinada.

El acceso a un determinado capital subcultural se produce fundamentalmente mediante el contacto con otras personas, y en este sentido está ligado a lo que se ha denominado capital social (Portes, 1998). Capital social y capital cultural están relacionados porque el capital social, además de proporcionar recursos de manera directa es la vía para la aculturación y, por lo tanto, para la obtención de capital cultural. Distinguimos dos tipos de capital social (Putnam, 2007): el capital social vínculo que deriva del contacto con personas del propio grupo étnico, o de grupos con contenido étnico similar, y el capital social puente, que se genera al ponerse en contacto con personas de otros grupos étnicos. El segundo tipo de capital cultural da acceso a repertorios culturales nuevos y puede dar lugar a procesos de aculturación.

Los individuos ponen en marcha estrategias en el campo social. Estas estrategias les permiten adquirir y poner en juego diferentes tipos de capital cultural y capital social a través de las interacciones con personas de diferentes grupos. Los diferentes capitales culturales pueden ser puestos en juego en diferentes campos y proporcionan acceso a recursos de tipo económico, estatus, apoyo emocional, etc. Consideramos que las estrategias están orientadas a la satisfacción de necesidades y que la satisfacción de necesidades está mediada por satisfactores (Max-Neef, 1993). En ocasiones los individuos ven su acción orientada por satisfactores violadores o destructores, que tienen un efecto negativo en la satisfacción de necesidades y que aparecen ligados a las necesidades de protección y al miedo (Max-Neef, 1993, pp. 57-65). Denominaremos contracapitales a los polos de capital cultural que giran en torno a estos satisfactores destructores. La obtención de un contracapital genera reconocimiento en el seno del grupo; pero lejos de proporcionar un capital que dé acceso a recursos, el contracapital suele llevar a una mayor discriminación y un mayor estigma.

Tenemos, por lo tanto, un campo étnico con diferentes grupos que generan diferentes capitales, algunos de los cuales, los contracapitales, tienen un efecto negativo en la satisfacción de necesidades. Los inmigrantes tienen que definir su modo de incorporación a través de estrategias basadas en la obtención de capital cultural y capital social. Cuando las estrategias de los individuos están centradas en el contacto con personas del propio grupo étnico (capital social vínculo) estaríamos ante estrategias de repliegue étnico. Por el contrario, cuando se centran en el contacto con personas de otros grupos étnicos (capital social puente) se trataría de estrategias que implican algún tipo de asimilación. En la literatura reciente se han identificado diferentes tipos de asimilación. En este sentido, la teoría de la asimilación segmentada distingue entre la asimilación tradicional, en la que se consigue una movilidad social ascendente, y la asimilación a la baja, que implica una movilidad social descendente mediante el acercamiento a los grupos excluidos de la sociedad de recepción (Portes y Zhou, 1993).

Resumiendo, los inmigrantes deben definir sus procesos de incorporación en un campo social, el campo étnico, en el que existen diferentes grupos que son capaces de definir subcampos étnicos diferenciados; es decir, los grupos definen identidades diferenciadas y la pertenencia a dichos grupos, la adhesión a las pautas identitarias de los mismos, proporciona un determinado capital cultural. Este capital cultural da acceso a diferentes tipos de recursos y a la satisfacción necesidades. Los inmigrantes deben definir sus estrategias de acumulación de capital, y en última instancia su modo de incorporación, en base a la obtención de los diferentes tipos de capital presentes en el campo étnico. 


\section{METODOLOGÍA}

Desde el punto de vista metodológico, se ha seguido el procedimiento de generalización analítica propuesto por Yin $(2003$, p. 3), en la que, a partir de una serie de casos empíricos concretos, se generaliza a una teoría y no a una población, como en el caso de las generalizaciones de tipo cualitativo.

A partir de la información obtenida en las entrevistas se han seguido dos procedimientos para reconstruir la estructura del campo étnico en el que operan los jóvenes marroquíes: la identificación de los diferentes polos de capital, es decir, los diferentes subcampos ligados a los diferentes grupos; y el análisis de las diferentes estrategias de acumulación de capital que pueden poner en marcha los jóvenes marroquíes

Distinguimos dos tipos de polos de capital, aquellos a los que se accede a través del capital social vínculo; es decir, a través de lazos con los miembros del propio grupo o con miembros de grupos con contenidos culturales similares; y, por otro lado, a los que se accede a través de capital social puente y dan acceso a capitales culturales ajenos a los del propio grupo. Cada polo debe ser definido en base al grupo social que lo crea, al contenido cultural en el que se fundamenta y en base a los recursos a los que da acceso.

En segundo lugar, basándonos en la distinción entre los dos tipos de polos de capital presentamos dos tipos ideales de estrategias de acumulación de capital social y cultural (el repliegue étnico y la asimilación), y analizamos los resultados que ofrecen en términos de acumulación de capital y acceso a recursos. Veremos que, en este caso, las más fáciles de poner en práctica y las que ofrecen unos recursos más inmediatos y seguros son las estrategias basadas en el repliegue étnico.

El principal instrumento de recogida de información ha sido la elaboración de una serie de entrevistas en profundidad realizadas entre 2011 y 2014. Se han realizado 25 entrevistas a jóvenes marroquíes residentes en Bizkaia, mayores de edad, pero que han estado acogidos en los centros de menores de la provincia en los años previos a la realización del estudio. En el momento de realizar las entrevistas, los jóvenes entrevistados eran usuarios del programa de emancipación Mundutik Mundura de la Diputación Foral de Bizkaia y se encontraban en un proceso de inserción social tutelado por educadores sociales.

También se han realizado entrevistas a profesionales y miembros de asociaciones: educadores, responsables de centros y asociaciones, profesores de enseñanza reglada o representantes de mezquitas.

La metodología utilizada al hacer las entrevistas ha sido cualitativa y semiestructurada partiendo de un guion, pero dejando hablar libremente a los entrevistados. Algunas entrevistas se han desarrollado a lo largo de una única sesión mientras que, en otros casos, se han prolongado a lo largo de diferentes sesiones. También se ha recogido información en diferentes entornos informales.

\section{RESULTADOS}

Hemos dividido la exposición de los resultados en tres partes. En la primera se exponen los diferentes polos de capital. En la segunda, se expone gráficamente la estructura del campo étnico en el que tienen que definir sus estrategias los jóvenes marroquíes en Bizkaia. En el tercer apartado se analizan dos tipos de estrategias de acumulación de capital en el campo: el repliegue étnico y la asimilación. 


\subsection{Polos de capital}

Una de las evidencias que apareció inmediatamente a lo largo de las entrevistas es la situación de aislamiento social de los menores marroquíes durante la etapa de tutela. Con la mayoría de edad, llega la emancipación y, en teoría, existe una mayor libertad de movimiento; sin embargo, la posibilidad de establecer contacto con población normalizada sigue siendo muy reducida. Los jóvenes marroquíes apenas comparten espacios con la población autóctona; son discriminados en el acceso a muchos espacios de socialización públicos (bares y discotecas, centros comerciales) y son controlados e identificados habitualmente por la policía.

A lo largo de las entrevistas se han identificado siete polos de capital consistentes. Los cuatro primeros están basados en el capital social vínculo, es decir, surgen de contactos entre miembros del mismo grupo étnico o de grupos con los que se comparte algún contenido cultural relevante: el grupo de jóvenes marroquíes, los jóvenes marroquíes con problemas de disciplina, la familia en Marruecos y las comunidades islámicas de Bizkaia.

A pesar de que los jóvenes marroquíes tienen, en general, pocas posibilidades de establecer relaciones con población autóctona, se han identificado dos grupos con los que los jóvenes marroquíes tienen contacto y que constituirían polos basados en el capital social puente: el de los jóvenes autóctonos y el de los educadores sociales.

Pasamos a continuación a describir los polos en base al grupo que los sustenta, el contenido cultural específico que los define y los recursos que proporcionan.

\subsubsection{Polo 1: "los chicos"}

Los jóvenes marroquíes suelen hacer referencia a sus iguales como "los chicos". Este grupo social va a constituir una importante red de apoyo para los jóvenes.

...de manera que si uno se ha quedado fuera del albergue, entre ellos también hay

una red solidaria maja, acogen porque es amigo o amigo de un amigo (Educadora Programa Hemen).

El estar bien conectado con otros jóvenes marroquíes se revela como un factor esencial para hacer frente a situaciones de desamparo. Por un lado, puede facilitar acceder a empleos.

Cuando terminó el curso, tenía un amigo que trabaja en una empresa y le ha oído que le falta alguno y le ha dicho que falta un chico, que si conoces a algún chico y me ha llamado y me ha dicho que tengo un trabajo. (Joven marroquí 1).

Tener amigos también sirve para conseguir recursos económicos en momentos de vulnerabilidad.

Si tienes un amigo que está jodido tienes que ayudarle, dejándole dinero. Como ahora yo (...) estoy jodido mis amigos me llaman, me dan 5 euros, me dejan dormir en sus casas (Joven marroquí 6).

El acceso a la vivienda es unos de los problemas más acuciantes de los jóvenes marroquíes. Muchos caen en la indigencia. Para hacer frente a esta situación, el estar bien conectado con el grupo de "los chicos" es fundamental. A continuación se reproduce el discurso de un joven que tiene las relaciones casi rotas con el colectivo de los jóvenes marroquíes. Se encuentra en un piso de una asociación dentro del programa de emancipación Mundutik Mundura:

Los chicos meten a amigos que están en la calle. Solo puedes meter a dos personas y meten a quinientas personas... Suelen decir. "Yo ayudo para que si yo necesito me ayuden". Pero a no ser que seas amigo no te llevan a casa (Joven marroquí 5).

Respecto al contenido cultural concreto de este polo de capital cultural, habría que 
apuntar que la identidad de los chicos sobre la que se crea el polo de capital subcultural se forma, en buena parte, en contraposición a la de los jóvenes autóctonos. Se daría aquí lo que Bauman y Gingrich denominan la gramática de la orientalización (Baumann y Gingrich, 2006). En este discurso un joven relaciona la cultura de los jóvenes autóctonos con el estereotipo de joven que aparece en el programa "Hermano mayor".

A mi me gustan las noticias y el programa de "Hermano mayor" pero los padres me dan mucha pena. El niño cuando tiene 3, 4 años, le tratan muy bien, al final sale torcido. Un día he visto a una chica que empuja a su madre, me da mucha pena. Allí, mi madre viene a la mañana, y le pongo una sonrisa. Aquí los jóvenes no tratan bien a sus padres. En Marruecos, no. (Joven marroquí 15).

La identidad de los chicos gira también en torno a preocuparse más por las necesidades del resto de miembros del grupo que por los lujos propios:

Hay que vestir ropa buena y limpia, pero hay algunos que pasan. Hay gente que compra zapatillas de 120 pero ¿para qué voy a comprar? Compro unas de 30, 40 y si tengo mucha pasta, doy al que no tiene para comer (Joven marroquí 15).

\subsubsection{Polo 2: "los malos", dinámicas de contracapital en los centros}

Este polo es problemático como concepto. Aunque lo hemos incluido entre los polos de capital basados en el capital social vínculo, porque es un polo que se forma en el seno del grupo de jóvenes marroquíes, en realidad es también, en gran medida, consecuencia de estrategias basadas en el capital social puente, concretamente de estrategias de asimilación fallidas o lo que se ha denominado asimilación a la baja (Portes y Zhou, 1993). Como veremos más adelante, este polo aparece conectado al polo de los jóvenes autóctonos.

Aunque de ninguna manera puede generalizarse, existen ciertos menores que presentan graves problemas de conducta y que incumplen sistemáticamente las normas de los centros, generando conflictos que, en ocasiones, puede llegar a ser graves. Los menores que incumplen sistemáticamente las normas constituirían un polo de capital cultural que denominaremos "los malos". Agruparse en torno a este polo provoca en los jóvenes numerosos problemas y agudiza la situación de fragilidad. Por ello categorizaremos este polo como un polo de contracapital. Daría acceso a satisfactores violadores relacionados con el miedo y el sentido de pertenencia.

Como apunta esta educadora el fracaso en los procesos educativos está ligado en algunos casos a la existencia de problemas familiares, psicológicos y de consumos previos al proceso migratorio.

Los que fracasan es porque tienen problemas añadidos. (...) consumos o trastornos mentales, componentes delictivos, etc. Estos factores suelen estar más unidos con chavales del Norte de Marruecos, que han estado mucho tiempo en Tánger o en Casablanca en ciudades grandes (Educadora Programa Hemen).

Las compañías en los centros y fuera de ellos van a influir de manera importante en el comportamiento de los jóvenes y en la formación de grupos de chavales que enfrentan las normas de los centros, consumen drogas y .

Si quieres tener una vida buena, tienes que buscar unos amigos buenos, que te enseñen cosas buenas, que te ayuden. Si estoy con un amigo que respeta a otra gente, si voy a estar con él, siempre voy a estar bien. Si voy con otro que roba, yo voy a robar (Joven marroquí 15).

Algunos jóvenes vinculan la relación con menores autóctonos, que puede comenzar en los centros educativos, con el principio de comportamientos negativos. El hecho de que la familia se encuentre lejos y no puede realizar ese función fiscalizadora del comportamiento 
y el contexto con una cultura diferente que es más laxa en lo relativo a los consumos de drogas es situado por algunos menores en el comienzo de las conductas disruptivas.

Algunos hacen como los chicos de aquí. Llevan pendientes, salen con las chicas, van de fiesta, empiezan a beber alcohol, fumar... En Marruecos están tus padres. Si te pillan fumando, te rompen la cara, pero aquí no, tus padres no están, puedes hacer lo que quieras (Joven marroquí 14).

Los jóvenes marroquíes relacionan en sus discursos las compañías con los comportamientos disruptivos. Mientras que el contacto con la población autóctona y con el que denominamos el grupo de "los malos" se considera que lleva a los jóvenes a malos comportamientos; el grupo de los chicos, los jóvenes marroquíes fieles a su cultura de origen, se toma como una influencia positiva.

A mí también me ha pasado eso. Antes salía con unos amigos, que fuman, van con chicas. Yo también he estado fumando. Eran marroquís y españoles (Joven marroquí 14).

Existen, por lo tanto, dos subpolos de capital dentro del grupo de los jóvenes marroquíes. El primero que describimos, al que denominábamos los chicos parece más ligado a la cultura marroquí de origen. Por el contrario el segundo, que denominaremos el de "los malos" aparece más ligado a los jóvenes autóctonos. Este segundo polo y las prácticas asociadas a él pueden tener efectos muy negativos en las condiciones materiales de los jóvenes marroquíes.

En primer lugar, el salir de fiesta y no respetar lo horarios supone una contravención de las normas de los centros de acogida. Esto puede dar lugar a que se les impongan medidas disciplinarias que posteriormente pueden suponer una limitación para acceder a los programas de emancipación como mayores de edad.

Yo tengo que salir, tengo que echar novia... hay que abrirse un poco. . . el sábado no me dieron paga. Dos semanas más sin paga. Me mandó salir del despacho y yo le decía que no, y allí me empujó, perdí el control y yo llorando y pegando. Luego vino el vigilante, y pegando al vigilante, dos educadores más y pegando a cinco. Me pusieron las esposas (Joven marroquí 16).

En caso de comportamientos que se adentran en lo delictivo los jóvenes pierden la posibilidad de renovar papeles, el acceso a programas, los empadronamientos y las ayudas.

La gente que conozco roba y todo, está en la calle, no tiene dónde comer, no tiene dónde dormir, van de comedores. Pero llegará un día en que no vas a tener ni albergue, ni comedor, ni nada. (Joven marroquí 16),

Además de perder el apoyo institucional los jóvenes que tienen este tipo de conductas pierden también el apoyo de la red de chicos marroquíes que era muy útil para hacer frente a situaciones de desprotección extrema como vimos anteriormente.

Por estas razones, podemos a afirmar que el polo de capital que denominábamos "Los malos" constituye en realidad un polo de contracapital que aumenta el riesgo de caer en una situación de desamparo. En este sentido, esta subcultura proporcionaría lo que Max-Neef denomina pseudo-satisfactores o satisfactores violadores que, a pesar de que parecen estar orientados a satisfacer una necesidad de identidad y protección, en realidad dificultan al individuo la satisfacción de sus necesidades.

\subsubsection{Polo 3: "la familia” en Marruecos}

Hemos incluido la familia como un capital social vínculo porque representa una conexión con la identidad originaria de los jóvenes, un lazo con su país de origen. El contacto con la familia suele ser frecuente a través, bien del teléfono, bien de internet. La familia es, en muchos casos, un elemento central en la definición del proyecto migratorio. La propia 
decisión de migrar se produce en el caso de muchos menores con el apoyo de la familia y forma parte de una estrategia familiar.

Hablar con la familia proporciona cierta satisfacción de las necesidades emocionales de los menores. Aunque la distancia también influye en este caso y muchos jóvenes eluden comentar con sus familiares los problemas para evitarles una preocupación.

Mi familia sabe que vivo en un piso, que tengo un educador. No entienden lo que

es. Les preocupa que esté bien. Si me pasa algo malo, no se lo cuento porque se van a preocupar más (Joven marroquí 10).

La familia se convierte también en ocasiones en una fuente de presión, ya que muchos de estos menores migran como parte de un proyecto familiar y en muchas ocasiones se convierten en la esperanza que tiene la familia para acceder a recursos económicos y poder mejorar sus condiciones materiales de vida.

Son el faro de la familia, si ellos fracasan, la familia fracasa y eso es una presión enorme (Educadora Programa Hemen).

El hecho de que la familia desconozca el contexto en el que se están desenvolviendo los jóvenes, dificulta su entendimiento de las situaciones a las que se ven sometidos y, en ocasiones, puede conducir a un exceso de presión sobre los jóvenes emigrantes en los que se ha depositado la esperanza de una mejora para el conjunto de la familia. Esto es especialmente importante en el caso de los jóvenes cuyo proyecto migratorio forma parte de una estrategia familiar.

Algunos vienen con 8.000 euros. Sus padres han tenido que pedir créditos de sus amigos. Pero si tienen un trabajo con 300 euros, a ese chico no le llega ni para comer, ni para pagar interés. Al final el chico, si manda, está jodido, Si no manda, siempre sus padres le dicen lo mismo: "tienes que mandar dinero". El chico está pensando todos los días "tienes que mandar dinero" (Joven marroquí 6).

En el caso de los programas de emancipación, en ocasiones, la necesidad de ayudar económicamente a la familia ante una situación de necesidad o simplemente de aportar un cantidad de dinero que haga ver a la familia que el proceso migratorio va por buen camino, puede entrar en contradicción con el funcionamiento de los programas, una de cuyas normas es el ahorro y la prohibición de enviar dinero a Marruecos.

Si se detecta que un joven ha mandado dinero a su familia y no cumple con los

objetivos de ahorro se le puede expulsar del programa (Educadora Mundutik).

A pesar de eso, el vínculo con la familia puede suponer una influencia positiva porque, como comentábamos anteriormente, el mantenimiento de las costumbres religiosas de origen y la disciplina que estas suponen es un factor positivo para adaptarse a las normas de los centros y de los programas educativos.

Los que tenían más contacto con la familia querían aprovechar el tiempo y luego

había otros que estaban ahí por estar. (Profesor centro fracaso escolar 2).

\subsubsection{Polo 4: "la comunidad islámica"}

La totalidad de los jóvenes marroquíes residentes en Bizkaia con los que se ha hablado se consideran musulmanes y llevan a cabo algún tipo de práctica relacionada con esta religión. Sin embargo, existen diferencias sustanciales en cuanto a las prácticas religiosas y a la forma de entender el islam en la sociedad de recepción. Pueden distinguirse tres perfiles: no practicante, practicante pasivo y practicante activo. El practicante pasivo cumple con las obligaciones del islam en cuanto a las prohibiciones, ayuno en Ramadán, etc., pero no participa activamente en la vida de las mezquitas y no tiene más que una relación casual con las comunidades islámicas que se crean en torno a las mezquitas. El practicante activo, por 
el contrario, sí que tienen vínculos de capital social significativos con otras personas que acuden a la mezquita, y participa activamente en las dinámicas de la misma, colabora en las actividades, etc.

La práctica religiosa puede ayudar a los menores y jóvenes a adaptarse a las normas de los centros. El respeto a las prácticas del islam incluye la abstinencia respecto al consumo de drogas, el respeto hacia las normas, la disciplina, la necesidad de llevar una vida ordenada etc. En este sentido, puede ser más sencillo para un practicante activo o pasivo adaptarse mejor a las normas del centro que para un no practicante. La mayoría de los chicos con mala conducta dentro de los centros podrían ser colocados en la categoría de no practicantes.

En la mezquita, hay gente mayor, se sienta al lado tuyo y te hablan. "Eres joven, estás en un país que no es tu país, tienes que estudiar, tienes que respetar a la gente mayor de este país, no tienes que fumar, tienes que hacer cosas buenas". Los que roban no van a la mezquita (Joven marroquí 15).

El capital cultural que se genera en torno a la comunidad islámica se construye en gran medida por oposición a la cultura autóctona, lo que constituiría una gramática de orientalización (Baumann y Gingrich, 2006). En este sentido la población autóctona y, concretamente, los jóvenes autóctonos van a constituir esa referencia negativa sobre la que construir la identidad propia.

No puedes decir que eres musulmán si vas fumando y bebiendo. Yo considero que está muy lejos de la religión (Joven marroquí 2).

Cuando tenía 17 siempre iba con chicas de aquí pero al final cambié toda mi vida... estar hoy con una, luego con otra, eso se llama aprovechar. A mí me parece que no es normal. Eso trae enfermedades, trae niños sin padres... (Joven marroquí 14).

Desde la propia mezquita también se hace una interpretación del menor marroquí como potencialmente problemático y se entiende el islam como una herramienta para corregir las desviaciones.

Como han crecido en una cultura musulmana tienen lo que es el conocimiento básico del Islam que es un lenguaje [...] que se puede utilizar para corregir esos chavales, para enderezarles, en vez de ir por un camino equivocado, pues corregir su camino. Aqui las instituciones no entienden eso [...] que esos tienen unas pautas culturales diferentes a las pautas culturales de esos chavales, de aquí. (Portavoz de la mezquita).

Además de proporcionar un capital cultural que se adapta mejor a las dinámicas de los centros de acogida, la participación activa en la vida de las comunidades islámicas va a proporcionar a algunos jóvenes un acceso a recursos que no tienen los no practicantes e incluso los practicantes pasivos.

La mezquita pone en contacto a los jóvenes marroquíes con una población musulmana que suele presentar un mayor grado de asentamiento en la sociedad. A través de este capital social los jóvenes marroquíes pueden acceder a recursos económicos de diferente tipo.

Si haces cosas buenas, tienes cosas buenas. En la mezquita hay gente mayor que tiene negocios, te ayudan en lo que sea, si necesitas pasta, te da. (Joven marroquí 15).

El capital social que se crea en la mezquita puede servir en ocasiones para encontrar piso, alternativas de ocio o un empleo.

Yo vivo en un piso con cuatro chicos marroquíes, mayores y que están trabajando. Me enteré del piso a través de la mezquita (Joven marroquí 8).

...tenemos un grupo de deporte, tenemos un grupo de teatro, de enseñar árabe a 
los jóvenes, de enseñar castellano a los chicos y a las chicas, de enseñar incluso euskera. Tenemos un grupo de subir al monte. Tenemos también una red de tiendas de musulmanes donde a veces necesitan trabajadores, a veces y vienen a coger de la mezquita. A veces una persona necesita encontrar una habitación. Viene a la mezquita y le hace contactar con otra persona. Una persona no sabe cómo presentar sus papeles, pues también les ayuda. (Portavoz de la mezquita).

\subsubsection{Polo 5: "jóvenes autóctonos"}

El joven autóctono es un contrapunto para la definición de la identidad de muchos jóvenes marroquíes. En este sentido, constituye un polo de capital diferenciado y el contacto con jóvenes autóctonos puede ser considerado como una forma de capital social puente, ya que da acceso a un capital cultural de un polo diferenciado del propio. En las entrevistas se han identificado tres fuentes principales de contacto con jóvenes autóctonos: los centros educativos PCPI, las parejas sentimentales y la participación en equipos deportivos.

El principal contacto de los MENAs con la población autóctona se da a través de los centros educativos PCPI a los que acuden alumnos autóctonos que han fracasado en el sistema educativo ordinario. El contacto con el alumnado autóctono de los PCPI puede dar lugar a aprendizajes de pautas culturales de desafío a las normas. El alumnado autóctono de los PCPIs está constituido por alumnos que han fracasado en el sistema educativo normalizado y suelen ser parte de los que suele denominarse cultura contraescolar. El contacto en las aulas provoca, en ocasiones, que esta cultura contraescolar se extienda los alumnos marroquíes.

Los magrebies al principio de curso eran bastante respetuosos, yo creo que estaban un poco a la expectativa, luego cuando veían como se comportaban los autóctonos ellos se empezaban a desmadrar también a faltar al respeto.... Los fines de semana solían coincidir con los autóctonos en los mismos sitios de ocio. Ellos oían a los autóctonos hablar de los sitios e iban allí, pero cada uno con su grupo. .. Los chavales autóctonos también algunos tenían situaciones malas en casa y otros fingían un poco tenerlo, fingían provenir de un ambiente marginal. $Y$ eso que les afectaba a los magrebies porque su única referencia dentro de la sociedad autóctona eran estos chavales del CIP que se movían un poco por ambientes marginales. Yo creo que hubiera sido mejor que estudiaran con otro tipo de gente con más edad, con otros problemas y otros hábitos (Profesor centro orientado al fracaso escolar 2).

Un acercamiento al polo de capital de los jóvenes autóctonos puede suponer también una modificación en las expectativas de algunos jóvenes. A modo de ejemplo, una educadora relata el caso de un joven marroquí que se relaciona fundamentalmente con jóvenes autóctonos (la cuadrilla de su novia) y que acaba de rechazar un puesto de trabajo en condiciones precarias por no ajustarse a sus expectativas.

Rechazó el contrato en prácticas que le ofrecieron porque dice que le pagan muy poco. Está en casa sin hacer nada y cuando se le acaben las ayudas va a tener problemas. Les ofrecen condiciones muy malas, pero es la manera que tienen de poder conseguir un trabajo mejor. Muchos chicos han empezado con contratos en prácticas y luego les han renovado y están trabajando. (Educadora Mundutik Mundura).

El contacto con los jóvenes autóctonos también introduce unas pautas de consumo que, en el caso de los jóvenes marroquíes, resultan disfuncionales e insostenibles. Siguiendo con el caso anterior:

Quiere mantener un nivel de vida que no es real. Compra ropa cada dos por tres 
y está apuntado al gimnasio. Ha tenido que pedir prestado para pagar el alquiler del piso. Debe dinero a la gente y esto está provocando que muchos le dejen de hablar. (Educadora Mundutik Mundura).

Como influencia positiva del contacto con los jóvenes autóctonos se puede destacar el aprendizaje del lenguaje y un mejor conocimiento de la realidad cultural autóctona. Esta adquisición de capital cultural puede ser positiva para tener un mejor manejo del idioma y para generar cierta red entre las personas autóctonas. En cualquier caso y como hemos visto anteriormente, la red fundamental de apoyo material para los jóvenes marroquíes está constituida por personas inmigrantes.

Con la novia mejoré castellano. Me siento con ella y recordé los tiempos en que no sabía castellano.... A través de la novia conozco a sus padres, sus amigos [...] Estar con la novia te sirve para conocer gente. He conocido un montón en todo este tiempo. Me llaman para ir a la lonja. (Joven marroquí 4).

\subsubsection{Polo 6: Educadores}

Otro colectivo autóctono con el que los jóvenes marroquíes tienen un importante contacto es el de los educadores y profesores. El grueso de las relaciones de este tipo son relaciones de tipo profesional. Sin embargo, también se establecen relaciones personales que en algunos casos llegan a ser importantes. Puede ser, además, una fuente de capital social que da acceso a recursos económicos en situaciones de dificultad.

En la etapa de tutela y en etapas posteriores mantener una buena relación con los educadores es importante ya que son estos los que van a tomar muchas de las decisiones trascendentales en la trayectoria de los jóvenes marroquíes. Un ejemplo de estos los constituyen las ofertas de trabajo que llegan a las asociaciones. Son los educadores quienes deciden cuál de los chicos es más adecuado para desempeñar el puesto y hacen propuestas a tal efecto. Es el caso del Joven marroquí 1 que consigue un trabajo en una empresa de inserción. En la obtención de este puesto tiene bastante que ver la buena relación que ha tenido con los educadores, lo que provoca que le propongan como candidato. Los jóvenes marroquíes son perfectamente conscientes de esta circunstancia.

Desde Amorebieta todos los educadores confían en mí, me daban las llaves de la oficina, si quiero coger un papel algo. Han pasado tres años pero a veces me llaman

los de Amorebieta. Si respetamos a los educadores, te respetan y hablan de ti bien. (Joven marroquí 15).

Entre los educadores se percibe una preferencia por el tipo de usuario que cumple las normas y obedece las indicaciones que se le hacen desde el sistema de intervención. El perfil que mejor se acomodaba a los requisitos que impone el sistema de intervención es el que tiene las referencias en la cultura de Marruecos y está menos "contaminado" por las expectativas, gustos y patrones de vida de la cultura autóctona. Es decir, el que mejor se acomoda a su situación objetiva de vulnerabilidad, el que no se quiere asimilar, porque asimilarse supone exigir lo que exigiría un autóctono y, por tanto, más de lo que el sistema de intervención puede ofrecer a estos jóvenes.

Por los educadores están mejor vistos los que son como los de Marruecos (Joven marroquí 16).

En este sentido, aunque los educadores puedan constituir un polo de capital, la relación educador-educando es una educación de poder y tiende en muchos casos a mantener las diferencias más que a suprimirlas. La barrera cultural que existe tiende a mantenerse porque las interacciones educador-educando están orientadas a crear unas pautas culturales en el educando que no son las propias del educador, sino las que se suponen adecuadas para hacer 
frente a la situación desfavorable que el educando sufre. En este sentido, los contactos formales con los educadores son una fuente de capital cultural muy limitada.

Los contactos que pueden aportar quizás más recursos a los jóvenes marroquíes son los que surgen de relaciones informales, es decir, los que superan el marco educador-educando y se establecen entre personas. Sí que se dan, en este sentido, ayudas a nivel económico o emocional por parte de algunos educadores a aquellos jóvenes con los que tienen una relación afectiva más cercana. Estas relaciones pueden ser una fuente de contactos informales para conseguir vivienda o trabajo o incluso apoyos dinerarios en situaciones de necesidad.

Yo sí que he ayudado económicamente a chavales que tenían una necesidad y con los que he tenido buena relación. Es como si le pasa algo así a un amigo tuyo. Si tienes dinero y él tiene una necesidad, le ayudas. (Educadora Programa Mundutik Mundura).

\subsection{Mapa del campo étnico}

Explicados los diferentes polos de capital, la representación gráfica del campo sería la siguiente. Se trata de una representación de carácter intuitivo basada en los datos cualitativos extraídos de las entrevistas. Las deformaciones representan los polos de capital, las líneas las conexiones y el círculo la proximidad cultural que observábamos que existía entre la identidad "los chicos" y la de la comunidad musulmana. La familia ejerce su influencia desde la distancia a través del contacto por vía telemática.

Figura 1: campo étnico de los jóvenes marroquíes en Bizkaia

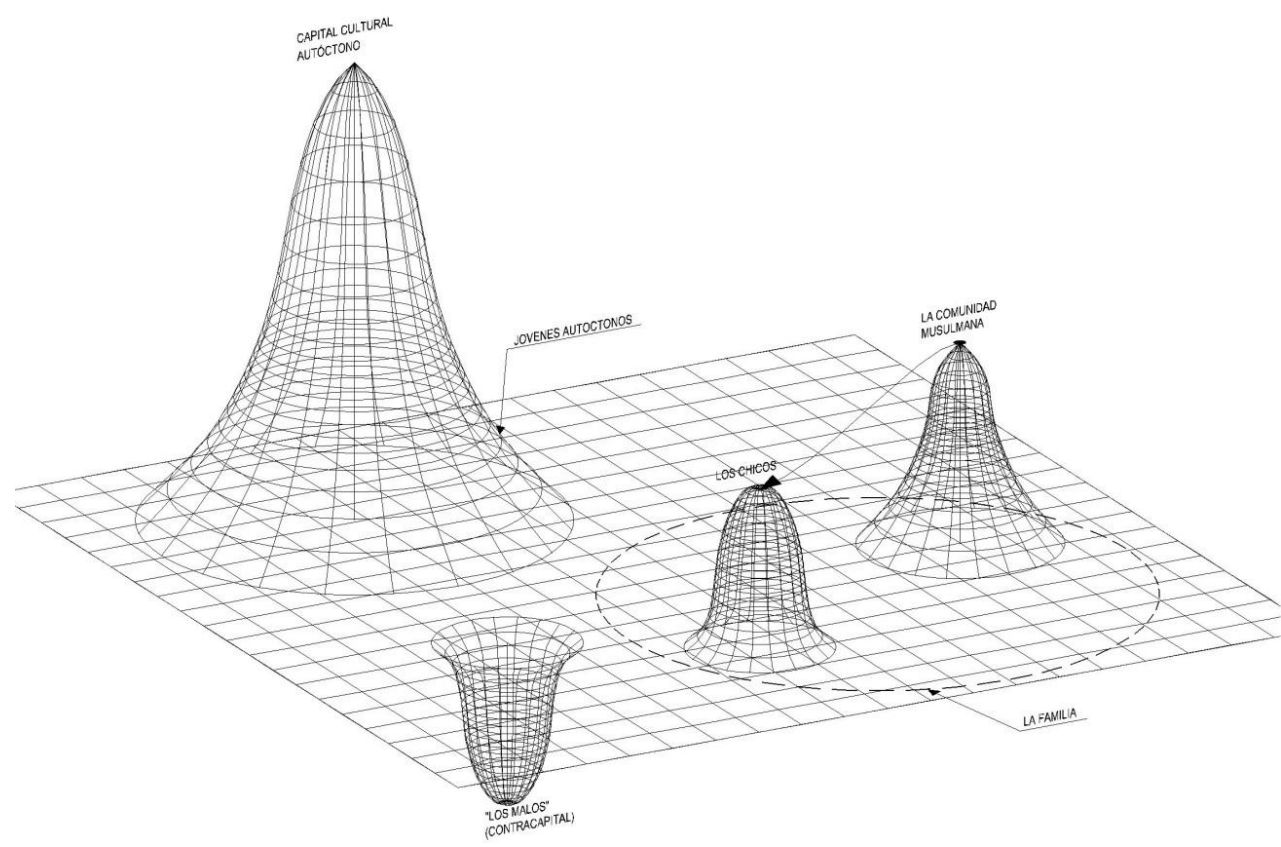

Fuente: Elaboración propia

En torno a cada polo de capital se darían un mayor número de conexiones y habría un 
mayor número de individuos. En terminología de redes sociales habría una mayor densidad.

Los polos de mayor altura ofrecen un mayor acceso a recursos y, por lo tanto, contribuyen más a la satisfacción de necesidades. Puede apreciarse como el polo de contracapital está deformado hacia abajo, lo que indica que contribuye negativamente a la satisfacción de necesidades.

Gráficamente se pueden interpretar las estrategias que describiremos a continuación de la siguiente manera. El repliegue étnico supondría quedarse dentro del círculo punteado y permanecer vinculado a la red de los chicos con la familia como sostén identitario y la comunidad islámica como soporte identitario y material. La estrategia de asimilación por su parte implica alejarse de ese círculo punteado y aproximarse al capital cultural autóctono, lo que conduce a una pérdida de acceso a recursos e incluso conlleva un mayor estigma o, incluso, la aproximación al grupo de contracapital "los malos".

\subsection{Estrategias de acumulación de capital: repliegue étnico frente a asimilación}

De cara a la interpretación de las trayectorias de los jóvenes inmigrantes en Bizkaia vamos a proponer dos estrategias típico-ideales. El primer tipo de estrategia se corresponde con lo que llamábamos estrategia colectiva-grupal o basada en el capital social vínculo y daría lugar al repliegue étnico. El segundo tipo sería una estrategia individual basada en el capital social puente y, de tener éxito, se aproximaría a lo que se define como asimilación.

Al comparar estos dos tipos de estrategia en términos del capital y del acceso a recursos que proporcionan podemos determinar cuál de ellas es más fácil de articular en la práctica.

\subsubsection{Estrategias basadas en el capital social vínculo: repliegue étnico}

Este tipo de estrategia está basada en fortalecer los lazos con el grupo propio, de manera que podría considerarse una estrategia de repliegue étnico. El grupo étnico propio proporciona una serie de recursos a los miembros que lo forman. En la tabla 1 se resumen los recursos que proporciona esta estrategia a través de los diferentes grupos y los aspectos negativos que presenta.

Vemos que, entre los recursos que proporciona el capital social vínculo, habría que destacar que existen muchas fuentes de apoyo material y económico. Esto es especialmente relevante en la etapa como mayores de edad en la que la mayoría de los jóvenes se encuentran en situación de vulnerabilidad o de desamparo.

En este sentido, resulta significativo que en las entrevistas se han identificado jóvenes que han reconducido sus estrategias de acumulación de capital, desde estrategias más basadas en el capital social puente a estrategias basadas en el capital social vínculo. La finalización de la etapa de estudios rompe en algunos casos el contacto que existía con los jóvenes autóctonos. Por otro lado, la falta de dinero para mantener prácticas de ocio similares a las de los jóvenes autóctonos y el cierre de muchos de los espacios sociales también provoca que los jóvenes pongan en marcha el repliegue étnico.

... algunos cuando empiezan a trabajar se buscan otros amigos que tienen dinero

y cuando ya no tienen nada se vuelven a los amigos antiguos. (Joven marroquí 6).

Vemos, por lo tanto, que la falta de dinero para satisfacer las necesidades mínimas y para relacionarse con la población autóctona en ambientes de ocio es uno de los factores relevantes que explican el repliegue hacia el grupo propio.

El contacto con la mezquita, que es menor durante la etapa de menores, se va haciendo más importante en la etapa emancipada. Por un lado, la mezquita es más accesible cuando se vive en un entorno urbano y no en los centros residenciales de menores. Por otro lado, la situación de desamparo y la existencia de una identidad musulmana hace que muchos 
jóvenes rellenen su tiempo libre acudiendo a la mezquita.

Tabla 1- Estrategias basadas en el capital social vínculo

\begin{tabular}{|c|c|c|c|}
\hline \multirow[t]{2}{*}{$\begin{array}{l}\text { Recursos que } \\
\text { proporcionan }\end{array}$} & $\begin{array}{l}\text { A través del grupo de } \\
\text { los chicos }\end{array}$ & $\begin{array}{l}\text { A través de la } \\
\text { familia }\end{array}$ & $\begin{array}{l}\text { A través de la } \\
\text { comunidad } \\
\text { islámica }\end{array}$ \\
\hline & $\begin{array}{l}\text { Ayudas económicas en } \\
\text { caso de necesidad. } \\
\text { Apoyo emocional, } \\
\text { acompañamiento en el } \\
\text { tiempo de ocio. } \\
\text { Acceso a la vivienda y } \\
\text { al alojamiento de } \\
\text { emergencia. } \\
\text { Identidad coherente con } \\
\text { el contexto. } \\
\text { Expectativas acordes a } \\
\text { la situación objetiva. }\end{array}$ & $\begin{array}{l}\text { Apoyo emocional. } \\
\text { Apoyo económico } \\
\text { en el caso de } \\
\text { familias de clase } \\
\text { media. } \\
\text { Soporte } \\
\text { identitario. }\end{array}$ & $\begin{array}{l}\text { Ayudas económicas. } \\
\text { Alimento en casos } \\
\text { de escasez extrema. } \\
\text { Contactos para } \\
\text { conseguir vivienda. } \\
\text { Identidad coherente } \\
\text { con el contexto. } \\
\text { Expectativas } \\
\text { acordes a la } \\
\text { situación objetiva }\end{array}$ \\
\hline $\begin{array}{l}\text { Aspectos } \\
\text { negativos }\end{array}$ & \multicolumn{3}{|c|}{$\begin{array}{l}\text { - Agudizan el aislamiento respecto al resto de la sociedad. } \\
\text { - Dificultan la adquisición de repertorios culturales autóctonos, de } \\
\text { aprendizaje de la cultura autóctona, y dificultan, por lo tanto, la } \\
\text { movilidad social ascendente asociada a la aculturación. } \\
\text { - Generan grupos sociales separados con referentes identitarios y } \\
\text { culturales muy distintos y, en ocasiones opuestos, a los del grupo } \\
\text { étnico dominante }\end{array}$} \\
\hline
\end{tabular}

Fuente: Elaboración propia

Tenemos, de esta manera, que en algunos jóvenes se va a producir un repliegue étnico cuando llegan a la mayoría de edad, empujados por la situación de desamparo y por los problemas a los que puede conducir una estrategia basada en la asimilación cuando no se tienen las necesidades básicas satisfechas. Este sería el caso del joven marroquí 14 que tras una etapa caracterizada por una aproximación a la cultura autóctona una serie de problemas de salud le llevan a abandonar su anterior estilo de vida y amistades y a convertirse en un asistente asiduo a la mezquita de su barrio.

A mí también me ha pasado eso. Antes salía con unos amigos, que fuman, van con chicas. Yo también he estado fumando. Eran marroquís y españoles. ...Nadie sabe cuándo va a morir. Yo no sé lo que me va a pasar, pero Dios sí. Lo más importante es que tienes que creer en Dios y en Mahoma. Si estás fumando y te pegan, no tienes que decir me pegan, tienes que decir "me pegan para que deje de fumar. (Joven marroquí 14).

\subsubsection{Estrategias basadas en el capital social puente y capital cultural autóctono: asimilación}

Las estrategias basadas en el capital social puente pasan por una aproximación a la cultura autóctona, fundamentalmente a través del contacto con jóvenes autóctonos y con educadores. Los efectos de este tipo de estrategia pueden apreciarse en la Tabla 2. 
Tabla 2- Estrategias basadas en el capital social puente

\begin{tabular}{|l|l|l|}
\hline $\begin{array}{l}\text { Recursos que } \\
\text { proporcionan }\end{array}$ & $\begin{array}{l}\text { A través de los jóvenes } \\
\text { autóctonos }\end{array}$ & A través de los educadores \\
\cline { 2 - 3 } & $\begin{array}{l}\text { Aprendizaje del idioma } \\
\text { Conocimiento de las normas } \\
\text { culturales autóctonas que } \\
\text { faciliten una mejor acomodación } \\
\text { social. }\end{array}$ & $\begin{array}{l}\text { Aprendizaje del idioma } \\
\text { Conocimiento de las normas } \\
\text { culturales autóctonas que faciliten } \\
\text { una mejor acomodación social. } \\
\text { Acceso a recursos materiales (en } \\
\text { caso de establecerse una relación } \\
\text { personal) }\end{array}$ \\
\hline nspectos & $\begin{array}{l}\text { - Proporciona pocos recursos económicos, puede ser negativo en caso de } \\
\text { que se sustituyan los lazos vínculo por lazos puente y se produzca una } \\
\text { apartamiento respecto al polo subcultural de los chicos. } \\
\text { - Puede modificar las expectativas en un sentido negativo, ya que las los } \\
\text { hábitos y la visión de la vida de los jóvenes autóctonos son acordes a una } \\
\text { situación económica muy distinta de la de los jóvenes marroquíes. } \\
\text { - Como comentábamos la aproximación al capital cultural autóctono } \\
\text { puede conducir en algunos casos a aproximarse también al polo de }\end{array}$ \\
& $\begin{array}{l}\text { contracapital que denominábamos "los malos". Este polo se caracteriza } \\
\text { por unos patrones culturales próximos, en algunos casos a los del joven } \\
\text { autóctono (comprar ropas de marca, salir de fiesta, consumo de drogas, } \\
\text { etc.) pero sufriendo un nivel de estigma aún mayor que el de la población } \\
\text { marroquí "no asimilada". }\end{array}$ \\
\hline
\end{tabular}

Fuente: Elaboración propia

El apartamiento del grupo propio como consecuencia del acercamiento a la población autóctona puede producirse por una mayor inversión de tiempo en este tipo de relaciones, pero también por un rechazo de la cultura de origen estigmatizada y la adopción de pautas culturales autóctonas que no son aceptadas por el grupo de origen.

Si yo tengo un amigo y lo conozco y se junta con chicos que hacen cosas bien, pues me parece bien. Pero si se junta con chicos de aquí y fuman marihuana y se emborrachan, eso me parece mal. Algunos hacen cosas para que les acepten. Comen cosas y se juntan con chicas de aquí, comen cerdo, beben alcohol. Si una persona te quiere, tiene que aceptarte cómo eres. No tienes que cambiar para quererte. (Joven marroquí 6).

En la práctica existen chicos que compatibilizan los dos tipos de relaciones como el joven marroquí 2 que tiene un contrato regular, comparte piso con marroquíes pero cuya cuadrilla está constituida por chavales autóctonos a los que conoció a través del equipo de fútbol en el que jugaba. Otro ejemplo es el joven marroquí 18 que también compatibiliza relaciones intensas con sus compatriotas y está integrado en una cuadrilla autóctona. En ambos casos se trata de jóvenes con un empleo estable y las necesidades materiales resueltas.

Por otro lado, los jóvenes que apuestan más claramente por una estrategia basada en la asimilación, en el capital social puente y que, por lo tanto, se apartan más del grupo étnicos de los jóvenes marroquíes son los que, en caso de no tener un empleo estable, tienen una mayor probabilidad de sufrir situaciones de desamparo o de profundo desarraigo identitario. Serían casos de asimilación fallida. 


\section{CONCLUSIONES}

El análisis del campo étnico se muestra como una herramienta útil para estudiar las posibilidades de incorporación de los inmigrantes a las sociedades de acogida, por estudiar conjuntamente el capital social, es decir las posibilidades de contacto con diferentes grupos, y el capital cultural, que nos muestras como se construyen las identidades, la diferencias y como estas se convierten en desigualdades.

El análisis del campo étnico en el que se desenvuelven los jóvenes marroquíes en Bizkaia nos ha mostrado que las estrategias de repliegue étnico ofrecen un mayor acceso a recursos que las estrategias de asimilación. Las estrategias de asimilación exponen a los jóvenes marroquíes a una mayor fragilidad y pueden dar lugar a situaciones de asimilación a la baja como el caso del polo de contracapital "los malos".

Las estrategias basadas en algún grado de asimilación y en el contacto con población autóctona sólo pueden tener éxito en jóvenes con empleos estables que les permiten tener las necesidades materiales cubiertas. Por el contrario, las situaciones de desamparo o fragilidad incentivan el repliegue étnico. Vemos, por lo tanto, que la integración de las minorías étnicas es complicada en ausencia de un sistema económico que garantice la satisfacción de las necesidades materiales.

Estos resultados son coherentes con trabajos como el de Granovetter (1973), que proponen que el capital social vínculo (lazos débiles en su terminología) juega un papel fundamental en situaciones de vulnerabilidad.

El estudio del campo étnico nos permite además conocer las razones estructurales, tanto materiales como culturales, de que se articulen unas u otras estrategias. De esta manera, podemos afirmar que fenómenos como el repliegue étnico o la asimilación son estructuralmente complejos y que no están condicionados únicamente por la mera voluntad de los inmigrantes o por políticas de carácter voluntarista que dicen buscar la integración sin actuar sobre las causas profundas de los procesos sociales.

\section{BIBLIOGRAFÍA}

Aranguren, M. R. (2011). “Aquí no entran negros ni moros”. Pikara magazine, en https://www.pikaramagazine.com/2011/03/aqui-no/

Ararteko. (2005). Situación de los Menores Extranjeros No Acompañados en la CAPV: Informe Extraordinario del Ararteko al Gobierno Vasco. http://www.ararteko.net/RecursosWeb/DOCUMENTOS/1/1_10_3.pdf

Ararteko. (2011). Infancias vulnerables. Informe Extraordinario del Ararteko al Gobierno Vasco, en http://www.ararteko.net/RecursosWeb/DOCUMENTOS/1/1_2354_3.pdf

Barba del Horno, M. (2019). "Los menores marroquíes en Bizkaia. Un colectivo en cuarentena social". Inguruak. Revista vasca de sociología y ciencia política, 67, 1-21.

Barth, F. (1976). Los grupos étnicos y sus fronteras: la organización social de las diferencias culturales. Fondo de cultura económica.

Baumann, G. y Gingrich, A. (2006). Grammars of identity/alterity: a structural approach. Berghahn Books.

Belmonte, J. L., Meneses, E. L., Cano, E. V., y Cabrera, A. F. (2019). “Avanzando hacia la inclusión intercultural: Percepciones de los menores extranjeros no acompañados de centros educativos españoles". Revista de Educación Inclusiva, 12(1), 331-350.

Bourdieu, P. (1988). La distinción: criterios y bases sociales del gusto. Taurus.

Bourdieu, P. (1997). Razones prácticas. Sobre la teoría de la acción. Anagrama. 
Bourdieu, P. (2005). Una invitación a la sociología reflexiva (1a ed.). Buenos Aires: Siglo XXI Editores.

Bourdieu. (1986). "The forms of capital". En J.G. Richardson. Handbook of Theory and Research for the Sociology of Education. Westport, CT: Greenwood, pp. 241-258, http://www.socialcapitalgateway.org/content/paper/bourdieu-p-1986-forms-capital-richardson-jhandbook-theory-and-research-sociology-educ

Carter, P. L. (2003). "Black Cultural Capital, Status Positioning, and Schooling Conflicts for LowIncome African American Youth". Social Problems, 50(1), 136-155.

Díaz, B. y Fantova, J. (1998). El color de la sospecha. El maltrato policial a personas inmigrantes en el barrio de San Francisco (Bilbao). Bilbao: Likiniano Elkartea. http://centroderecursos.alboan.org/es/registros/1372-el-color-de-la

Fernández, B. y Barba del Horno, M. (2014). "Los jóvenes inmigrantes marroquíes en el sistema educativo de Bizkaia: diferentes inserciones y sus efectos en términos de capital social". Inguruak. Revista vasca de sociología y ciencia política, 57-58, 2595-2606.

Fernández, M., Valbuena, C. y Caro, R. (2017). Evolución del racismo, la xenofobia y otras formas de intolerancia en España. Informe- encuesta 2017. Observatorio Español del Racismo y la Xenofobia, http://www.mitramiss.gob.es/oberaxe/ficheros/documentos/Informe-Racismo-2017.pdf

Gozalo, A., Jiménez, E. y Vozmediano, L. (2010). ¿Menores o extranjeros? Análisis de las políticas de intervención sobre menores extranjeros no acompañados. Publicación del Ararteko, http://www.ararteko.net/RecursosWeb/DOCUMENTOS/1/1 2226 3.pdf

Granovetter, M. S. (1973). The Strength of Weak Ties. The American Journal of Sociology, 78(6), $1360-1380$.

Ikuspegi. (2018). Barómetro 2018. Percepciones y actitudes hacia la inmigración extranjera. Ikuspegiak. Observatorio Vasco de la Inmigración. http://www.ikuspegi.eus/documentos/barometros/2018/bar_CAE_2018_CAS_web.pdf

Lajusticia, M. G. L. (2018). "La construcción de la identidad. Menores extranjeros no acompañados en contextos de protección". RES : Revista de Educación Social, 27, 71-92.

Lamont, M. y Lareau, A. (1988). "Cultural Capital: Allusions, Gaps and Glissandos in Recent Theoretical Developments". Sociological Theory, 6(2), 153-168.

Max-Neef, M. (1993). Desarrollo a escala humana. Barcelona: Icaria Antrazyt.

Palma-García, M. de las O., Ruiz Mosquera, A. C., y Vives González, C. L. (2019). "Jóvenes inmigrantes extutelados. El tránsito a la vida adulta de los menores extranjeros no acompañados en el caso español”. EHQUIDAD. Revista Internacional De Políticas De Bienestar Y Trabajo Social, (12), 31-52. https://doi.org/10.15257/ehquidad.2019.0009

Portes, A. (1998). "Social Capital: Its Origins and Applications in Modern Sociology". Annual Review of Sociology, 24(1), 1-24.

Portes, A. y Zhou, M. (1993). "The New Second Generation: Segmented Assimilation and Its Variants". The Annals of the American Academy of Political and Social Science, 530, 74-96.

Putnam, R. D. (2007). "E Pluribus Unum: Diversity and Community in the Twenty-first Century The 2006 Johan Skytte Prize Lecture”. Scandinavian Political Studies, 30(2), 137-174.

Quiroga, V., Alonso, A. y Soria, M. (2009). Sueños de bolsillo. Menores Migrantes No Acompañados en el País Vasco. Servicio Central de Publicaciones del Gobierno Vasco. http://www.surgam.org/articulos/510/Suenyos \%20de\%20bolsillo\%20Castellano.pdf

Setién, M. L. y Barcelo, F. (2007). "La atención a los MENAs en el País Vasco. Modelos de intervención y luces y sombras en el sistema de acogida". Actas del Coloqui Internacional: "La migración de Menores extranjeros no Acompañados en Europa. Poitiers 11-12 octubre 2007.

Yin, R. K. (2003). Applications of case study research. Thousand Oaks, CA: SAGE. 


\section{Breve currículo:}

\section{Mikel Barba del Horno}

Doctor en Estudios sobre Desarrollo por la Universidad del País Vasco. Mi línea principal de investigación está centrada en los procesos de incorporación de los inmigrantes y la formación de identidades en contextos interétnicos. He trabajado principalmente en torno al fenómeno de los Menores Extranjeros no Acompañados. 